\title{
Chronic exposure to organophosphates: background and clinical picture
}

\author{
Robert Davies, Ghouse Ahmed \& Tegwedd Freer
}

\section{Case study}

A male farmer in his forties from the Irish Republic was referred for an opinion on his mood swings and their possible relationship with organophosphate exposure. He had been regularly exposed to organophosphorus sheep dips for around 15 years prior to the assessment.

His personal history until he became ill, four years prior to assessment, was unremarkable. There was no previous psychiatric history or any of substance misuse. He was happily married and, although not well-off, had no financial difficulties. There was no family psychiatric history.

Approxiamtely 10 years prior to assessment, he began to develop flu-like symptoms following each dipping lasting 2-3 days but these were not in any sense incapacitating. Four years prior to interview he developed a particularly severe bout of this lasting $4-5$ days, characterised by disorientation, sweating, excessive salivation and diarrhoea and followed by a two-week period of apathy, depression, muscular aches and pains and fasciculation. Subsequently, there were two further such episodes and during this time the following symptom pattern developed.

His mood became markedly unstable with brief swings into both tearful depression and extreme irritability. These periods generally lasted for hours only, although against a background from time to time of more protracted periods of low-grade depression of mood. He also experienced several episodes of impulsive suicidal thinking and on one occasion 'came to' in his yard holding a loaded shotgun to his mouth. A variety of antidepressants were tried, but with no, or only transient benefit. Adverse effects were prominent at relatively low dose.

His short-term memory and attention became impaired, with him frequently getting lost and forgetting what he intended to do. He stopped reading completely, a pastime which he previously enjoyed, because of his inability to concentrate and retain the plot. His wife was obliged to take on most of the farm paperwork.

He became profoundly sensitive to the presence of perfumes and similar substances and also very sensitive to the effects of alcohol. Prior to becoming ill, he was a social drinker only, but subsequently small quantities of alcohol had such a great effect on him that his frequent state of inebriation led to his admission for detoxification. It was a very brief stay indeed since it became rapidly clear to the treating psychiatrist that there was no alcohol dependence problem. Subsequent to his discharge, he stopped drinking alcohol. He also became highly sensitive to the presence of low concentrations of organophosphates to the point that he was unable to go to sheep sales. He described one episode of muscular twitching, excessive salivation, headache and abdominal cramps when in the vicinity of a passing flock of newly-dipped sheep.

His handwriting deteriorated markedly in legibility, with numerous spelling mistakes that previously he would not have made. He subsequently developed a significant word-finding disorder. His ability to sustain muscular activity became seriously impaired, to the point that he was barely able to carry out the most basic chores on the farm.

On this basis, he was diagnosed as suffering from the neuropsychiatric effects of organophosphate exposure (Ahmed \& Davies, 1997).

Robert Davies is a consultant psychiatrist in Somerset (Rydon House Mental Health Unit, Cheddon Road, Taunton TA2 7AZ; tel 01823 333438). Ghouse Ahmed is a locum consultant psychiatrist and has been an Associate Specialist in Somerset since 1972. Tegwedd Freer is a consultant in adult and old age psychiatry at Exeter \& District Community Health Trust. All the authors have been involved in recent years in research into the neuropsychiatric effects of exposure to organophosphates. 
Approximately six months after assessment he shot himself.

At post-mortem, evidence of extensive peripheral neuropathy compatible with an organophosphate aetiology but unlike the picture of alcoholic polyneuropathy was found.

\section{Clinical presentation of chronic organophosphate toxicity}

Patients who are adversely affected by organophosphate exposure are likely to be referred to psychiatrists because of mood swings, suicidality, cognitive impairment and chronic fatigue. Occasionally, they may be referred in the context of significant episodes of rage or drunkenness leading to criminal proceedings.

The psychiatrist meeting such a patient for the first time may be bewildered by the variety of neuropsychiatric symptoms described and also manifestations of other multi-system pathology. It is likely that the patient will have been referred to physicians and surgeons previously for one or more of a variety of complaints - shortness of breath, cardiac problems, paraesthesiae and muscular fasciculations, gastric pain, urgency and frequency of micturition, dental problems (teeth crumbling or falling out) and osteoporotic fractures and collapses. There is growing evidence to associate all of these causally with organophosphate exposure.

\section{Mood disorder}

The mood swings experienced by organophosphate exposees are of relatively brief duration - minutes to hours, but in the case of low mood, days - and involve swings into depression on the one hand and irritability and anger on the other. Swings into elation do not appear to be a feature of organophosphate toxicity, although there is one anecdotal case in the literature (Bradwell, 1994). The mood swings are generally unpredictable and unrelated to circumstance - they have the characteristic 'out of the blue' quality of 'endogenous' depressive states, but without the persistence. In extreme cases, the mood lability can resemble that of a post-stroke victim with sudden onset of profuse tearfulness for seconds followed by rapid and complete recovery. It is instability of mood rather than a pervasive mood state, and swings into irritability and at times explosive aggression in the absence of elevation of mood, that distinguish this from functional affective disorders and cyclothymia.
It has the characteristics of an organic mood disorder, but may in moderate to severe cases be perceived by the psychiatrist as evidence of personality disorder - a history of fairly abrupt personality change confirmed by the patient's partner or other family member should clarify the assessment.

\section{Suicidal thinking and behaviour}

The characteristic picture is of impulsive suicidal thoughts that are out of the blue and may result in serious action being taken. Tractors may be put into full throttle and aimed at walls or ditches; shotguns may be taken from their cabinet, loaded, and placed into the mouth of the sufferer; or nooses may be tied and fastened to supports. The means chosen tend to be highly effective and lethal if carried through. The duration of suicidal thinking tends to be very short indeed and hence there are numerous survivors to tell the tale. Many such survivors deprive themselves of ready methods, for example, by surrendering their shotguns. Others, unfortunately (as the case report shows) do not.

Epidemiological work from Spain supports the link between organophosphate exposure and increased suicide rates (Parron et al, 1996), and this link has also been recognised by the Royal College of Physicians \& Royal College of Psychiatrists (1998). Additionally, the almost complete lack of protective measures taken by sprayers, and the heavy level of use, may contribute to the high rates of suicide, often involving organophosphate ingestion, in the developing world.

\section{Cognitive impairment}

The typical patient complains of difficulty in retaining recently registered information and in concentrating adequately to fill in forms and other similar tasks. Names of familiar people may temporarily be forgotten (this may, in fact, be a manifestation of language disorder). In more severe cases, the ability to perform sequential tasks may be seriously impaired through forgetting that the previous step has been completed. Sufferers may become lost in familiar surroundings, fail to recognise acquaintances, and be unable to follow plots in books or on television. The patient invariably complains of memory problems - confabulation is not a feature.

The full profile of organophosphate-induced cognitive impairment remains to be established, but it is noteworthy that Stephens et al (1995) found evidence of 'subtle' changes in otherwise asymptomatic farmers exposed to organophosphates, compared with quarry workers who were not. 
A small number of neuropsychological assessments for medicolegal purposes suggest fundamental impairment of central information processing and an IQ loss, primarily involving dominant hemispheric function, compared with the National Adult Reading Test predicted value. These findings are not in the public domain, but research currently in progress is aimed at evaluating these anecdotes.

\section{Chronic fatigue}

Chronic fatigue states are frequently reported by individuals who have been exposed long-term to organophosphates. The status of chronic fatigue as a psychiatric or indeed a physical symptom is disputed (Anonymous, 1996; Royal Colleges of Physicians, Psychiatrists \& General Practitioners, 1997). The United States Centers for Disease Control include organophosphates as a cause of chronic fatigue syndrome (CFS). The presence of fatigue, however, should not divert attention from the other problems associated with organophosphate exposure. In this group, a diagnosis of CFS is unhelpful and misleading.

\section{Other symptoms}

In 1997, Ahmed \& Davies described a neuropsychiatric syndrome to which they gave the acronym COPIND - chronic organophosphate-induced neuropsychiatric disorder. This comprised 10 symptoms (Box 1) and was based on the clinical interview of 33 patients referred to them, 26 of whom reported the features of this condition. More detailed descriptions of these are available in Ahmed \& Davies (1997). Chronic fatigue was not included so as not to mask the coherence of COPIND as a syndrome and blur the distinction between this and CFS.

On the basis of these, it was suggested that COPIND, as defined by Ahmed \& Davies, is a true and coherent syndrome related causally to chronic exposure to organophosphate pesticides, cardinal features of which are mood instability, suicidal thinking, cognitive impairment, language disorder and inability to sustain muscular power with 'biological markers' such as alcohol intolerance and olfactory hypersensitivity. The conclusion requires caution, as the number of patients was small, there was no control group, and several patients were excluded because of incomplete information.

However, Davies et al (1999), in a larger study, confirmed the coherence of COPIND as a syndrome and provided evidence of a causal link with organophosphate exposure.
An extensive review of the broader neurological effects of chronic organophosphate exposure can be found in Jamal (1997), in which Ahmed \& Davies' COPIND is designated 'COPIND 2'.

\section{Background}

Organophosphates were first synthesised in the 19 th century as chemical curiosities, but in the early

Box 1. The symptoms of chronic organophosphate-induced neuropsychiatric disorder (COPIND) (Ahmed \& Davies, 1997)

Exacerbation of dipper's flu

One or more episodes of severe flu-like symptoms lasting more than three days following exposure. May be accompanied by hypersalivation, abdominal cramps and diarrhoea

Personality change

Mood destabilisation - see text

Suicidal thinking

See text

Cognitive impairment

See text

Language disorder

Word-finding and expressive disorders

Less commonly, more profound disruption

Alcohol intolerance

Marked increase in the inebriating effect of alcohol; may also involve severe hangover and quasi-allergic effects

Heightened sense of smell

Typically to perfumes and scents; may be so severe as to make patients feel ill

Handwriting deterioration

Significant decrease in legibility worsening with length of text

Organophosphate-sensitive

Symptoms of dipper's flu triggered by extremely low concentrations of organophosphates

Decreased exercise tolerance

Normal initial muscle power, but inability to maintain it; not generalised weakness 
1920s the German chemist Gerhard Schrader noted that some of these substances were lethal to insects and began research into their development as insecticides. The potential of these substances for other uses was rapidly recognised by the German military, and alongside Schrader's insecticide research, work was also undertaken to develop them as military nerve agents. By 1939, considerable stockpiles of the first of these, Tabun, were available to the German armed forces. Mercifully they were not used.

The allies were developing their own insecticides, organochlorines, which were themselves the product of the German chemical industry and imported covertly, the first being dichlor-diphenyltrichloroethane (DDT). The use of DDT substantially cut down morbidity from arthropod-borne illnesses in all theatres during the Second World War, and in Naples in 1944 it stopped a potential epidemic of typhus in its tracks, leading Winston Churchill to refer to it as "that miraculous powder".

After World War II, DDT and other organochlorines were widely used as insecticides in agriculture and domestically. Organophosphates were also developed, particularly as these were much more effective than organochlorines on arthropods other than insects. In 1962, Carson published the book Silent Spring, which documents the dramatic drop in bird life secondary to the reduction in insect populations, brought about by widespread use of organochlorines. This, together with rapidly mounting evidence of accumulation particularly in marine species, led progressively to the withdrawal of organochlorines from agricultural use and their replacement by organophosphates.

In 1976, the UK Ministry of Agriculture, Fisheries \& Foods (MAFF) introduced compulsory dipping of sheep twice yearly to prevent fly strike and other insect infestations. Failure to comply with this policy resulted in quite severe penalties for the farmer, and this policy continued up until 1992.

Initially, those using these agents were recommended to take minimal precautions against exposure - soft rubber gloves (which disintegrated after brief use) and aprons. Progressively, the degree of protection recommended (but never enforced) was enhanced to the point that, for the average hillfarmer, these precautions were impossible physically to follow. There is substantial evidence in the occupational health medicine field to indicate that even these levels of protection were insufficient to prevent significant absorption.

Accordingly, there is a substantial population of sheep farmers who have absorbed organophosphates over a considerable period and in significant quantities. Organophosphates are used much more widely than just as sheep dips (and military nerve agents). Cattle farmers use them as pour-ons, arable farmers and market gardeners use them on crops and stored grain, and domestically they are found in fly strips, woodworm and similar treatments, pet flea preparations and, in the case of malathion, treatments for human arthropod infestation.

Organophosphate toxicity problems are therefore not restricted to sheep farmers - many other occupational groups are at potential risk, as are those who are domestically or environmentally exposed.

\section{Mechanisms of toxicity}

Organophosphates are potent anticholinesterases and, in the case of military nerve agents, minute quantities are sufficient to induce a cholinergic crisis leading to tetany, respiratory paralysis and death. Agricultural organophosphates in a concentrated form have similar capability if ingested, and over the years they have found a further role as suicide tools, particularly in the developing world - a recent and important paper (Eddleston et al, 1998) has drawn attention to the devastating effects that this practice has had on the availability of intensive care beds in Sri Lanka.

Organophosphates deactivate cholinesterase by attaching an alkylphosphate group to the hydroxyl group of a serine residue at the enzyme's

Box 2. Chronology

1930s - organophosphate pesticides and nerve agents first produced

1950s - UK Government Chief Scientist warns of dangers of chronic exposure; resulting ill health designated a 'prescribed disease' (C3)

1960s - organophosphates begin to supercede organochlorines as pesticides

1976 - Ministry of Agriculture, Fisheries \& Foods introduces twice-yearly compulsory sheep-dipping

1981 - Guidance Note MS17 (Health and Safety Executive) highlights risk of exposure and need for protective clothing

1997 - first successful personal injury action in UK involving neuropsychiatric damage from chronic exposure 
active site. The bond formed is strong and cannot be displaced by high concentrations of acetylcholineorganophosphates therefore are non-competitive inhibitors of cholinesterase in much the same way that older monoamine oxidase inhibitors are of monoamine oxidase. Recovery from such inhibition generally takes 10-14 days.

This mechanism of action, however, suggests that other systems may be as vulnerable as cholinesterase and there is increasing experimental evidence that this is indeed so. This evidence is discussed in outline by Davies (1995) and it seems likely that many of the neuropsychiatric symptoms seen in chronic exposees are not related to cholinesterase inhibition and but are the result of other mechanisms such as serotonin depletion (Ali et al, 1979) and interference with the inositol polyphosphate second messenger system (Samoilov et al, 1994).

The dramatic increase in understanding of neurobiology that has resulted from the 'decade of the brain' is revealing further candidate mechanisms and, profoundly worryingly, the possible implications of exposure for the foetus, and the child. These are only now beginning to be explored.

\section{Epidemiology}

There are, to date, no reliable estimates of the prevalence of COPIND, or of the likelihood of a chronic exposee developing significant psychiatric problems. Davies et al (1999) suggest that perhaps $10 \%$ of exposees are at risk, based on a $16 \%$ rate in respondents and a 5\% rate in all questionnaires sent out. This is, at best, guesswork, and much larger studies are required.

There are a number of hypotheses to explain this differential vulnerability that are beyond the scope of this article. There is an increasing literature, both on toxicity-enhancing mechanisms, for example, rates of oxidation of thion (low toxicity) organophosphates to oxons (high toxicity), and on detoxifying mechanisms such as paraoxonase. Genetic differences seem to be important, and some have been established (e.g. Yamasaki et al, 1997; Furlong et al, 1998).

\section{Summary}

This article is intended to draw the attention of psychiatrists to the potential of organophosphate exposure to produce psychiatric disorder in terms of mood swings and cognitive impairment, and in particular, the risk of suicidal behaviour and also violence.

A subsequent article will address the issues of diagnosis and management.

\section{References}

Anonymous (1996) Frustrating survey of chronic fatigue. Lancet, 348, 97.

Ahmed, G. M. \& Davies, D. R. (1997) Chronic organophosphate exposure: toward the definition of a neuropsychiatric syndrome. Journal of Nutritional and Environmental Medicine, 7, 169-176.

Ali, S. F., Hasan, M. \& Tariq, M. (1979) Levels of dopamine, norepinephrine and 5-hydroxy tryptamine in different regions of rat brain and spinal cord following chronic administration of the organophosphate pesticide diclorvos. Indian Journal of Experimental Biology, 17, 424426.

Bradwell, R. H. (1994) Psychiatric sequelae of organophosphate poisoning: a case study and review of the literature. Behavioural Neurology, 7, 117-122.

Carson, R. (1962) Silent Spring. London: Hamish Hamilton.

Davies, D. R. (1995) Organophosphates, affective disorders and suicide. Journal of Nutritional and Environmental Medicine, 5, 367-374.

--, Ahmed, G. M. \& Freer, T. (1999) Chronic organophosphate induced neuropsychiatric disorder (COPIND): results of two postal questionnaire surveys. Journal of Nutritional and Environmental Medicine, 9, 123-134.

Eddleston, M., Rezvi Sheriff, M. H. \& Hawton, K. (1998) Deliberate self harm in Sri Lanka; an overlooked tragedy in the developing world. British Medical Journal, 317, 133135

Furlong, C. E., Li, W. F., Costa, L.G., et al (1998) Genetically determined susceptibility to organophosphorus insecticides and nerve agents: developing a mouse model for the human PON 1 polymorphism. Biochemistry, 37, 237247.

Jamal, G. A. (1997) Neurological syndromes of organophosphorus compounds. Adverse Drug Reactions and Toxicology Reviews, 16, 133-170.

Parron, T., Hernandez, A. T. \& Villanueva, E. (1996) Increased risk of suicide with exposure to pesticides in an intensive agricultural area. A 12-year retrospective study. Forensic Science International, 79, 53-63.

Royal College of Physicians, Royal College of Psychiatrists \& Royal College of General Practitioners (1997) Chronic Fatigue Syndrome. Report of a Joint Working Group of the Royal Colleges of Physicians, Psychiatrists $\mathcal{E}$ General Practitioners. Council Report CR54. London: Royal College of Psychiatrists.

— \& Royal College of Psychiatrists (1998) Organophosphate Sheep Dip. Clinical Aspects of Long-Term Low-Dose Exposure. Report of a Joint Working Party of the Royal College of Physicians and Royal College of Psychiatrists. Council Report CR67. London: Royal College of Physicians.

Samoilov, M. O., Sofranov, G. A., Kamenev, A. L., et al (1994) Involvement of intracellular regulatory mechanisms in fosfacol effect of feline cerebral neurons (in Russian with English abstract). Eksperimental'naya Klinicheskaya Farmakologiya, 57, 13-16.

Stephens, R., Spurgeon, A., Calvert, I. A., et al (1995) Neuropsychological effects of long term exposure to organophosphates in sheep dip. Lancet, 345, 1135-1139.

Yamasaki, Y., Sakamoto, K., Watada, H., et al (1997) The Arg 192 isoform of paraoxonase with low sarinhydrolyzing activity is dominant in the Japanese. Human Genetics, 101, 67-68. 


\section{Multiple choice questions}

1. Organophosphates:

a are specific cholinesterase inhibitors

b inactivate cholinesterase by alkylphosphorylation

c were first synthesised in the 1930s

d are only used as pesticides

e are a major method of attempted suicide in developing countries.

2. Symptoms of chronic organophosphate-induced neuropsychiatric disorder include:

a persistent depression of mood

b anosmia

c attention and short-term memory impairment

d increased irritability

e alcohol intolerance.
3. Long-term exposure to organophosphates:

a invariably leads to COPIND

b may be associated with damage to systems other than the central nervous system

c is limited to farmers

d may be associated with episodic, flu-like illnesses

e may be associated with suicidal behaviour.

$\begin{array}{llllll}\text { 1 } & & 2 & & & \\ \text { a } & F & \text { a F } & \text { a } & \text { F } \\ \text { b } & T & \text { b F } & \text { b } & \text { T } \\ \text { c F } & \text { c T } & \text { c } & \text { F } \\ \text { d F } & \text { d T } & \text { d } & \text { T } \\ \text { e } & \text { T } & \text { e T } & \text { e } & \text { T }\end{array}$

\section{Assessing Forensic Mental Health Need: Policy, Theory and Research} Andrea Cohen and Nigel Eastman

Mentally disordered offenders (MDOs) have dominated the recent political agenda for mental health care emphasised by cases such as those of Christopher Clunis and Michael Stone. Yet successive governments have constrained the development of all health and social services by the requirement that they must be demonstrated as capable of benefiting those to whom they are applied.

This book analyses the development of government policy for services for MDOs since the Butler report in 1975 and provides a theoretical framework for adjudging research which purports to show a need for MDO services of various types. It also comprehensively reviews relevant empirical research and addresses the important links between need and outcome. Finally, it emphasises the subjective and value-laden nature of all needs assessments and their interpretation and poses the crucial question of whose need it is that we address through MDO services, those of the patient or of society. This book will be valuable to clinicians and researchers working in mental health services, policymakers, service commissioners and managers.

Andrea Cohen and Nigel Eastman work in the Mentally Disordered Offender Policy Research Unit at St George's Hospital Medical School.

May 2000, hardback, £30.00, ISBN 1901242420

Booksales, The Royal College of Psychiatrists, 17 Belgrave Square, London SW1X 8PG Telephone +44 (0)20 72352351 ext. 146; fax +44 (0)20 72451231

http://www.rcpsych.ac.uk 